www.conferenceie.ase.ro

\title{
A GRAPH DATABASE APPROACH TO MANAGING DATA WITH UNPREDICTABLE SCHEMA IN ACADEMIC INFORMATION SYSTEMS DEVELOPMENT
}

\author{
Ștefan UIFĂLEAN \\ Babeş-Bolyai University, Business Informatics Research Center, Cluj Napoca, Romania \\ suifalean@gmail.com \\ Gheorghe Cosmin SILAGHI \\ Babeş-Bolyai University, Business Informatics Research Center, Cluj Napoca, Romania \\ gheorghe.silaghi@econ.ubbcluj.ro \\ Robert Andrei BUCHMANN \\ Babeş-Bolyai University, Business Informatics Research Center, Cluj Napoca, Romania \\ robert.buchmann@econ.ubbcluj.ro
}

\begin{abstract}
Modelling information systems that are based on ever-evolving data, from both the size and the structure point of view proves to be a challenging software engineering undertaking. With a traditional relational database, any change in the data structure will propagate through the entire architecture of the software solution, requesting a lot of work both in the design and the implementation of the software product. With the advances of the graph databases, storing and using schema-less data that specifies relationships at instance level became a handy alternative. This paper presents a proof-of-concept solution for intuitively modelling interconnected elements and using them as a knowledge-base for an information system. By integrating a Neo4J store in a Spring Boot MVC application, we show how we can overcome the software engineering process problems when schema change requests are frequently propagated and how we can induce flexibility in both the data design and application development.
\end{abstract}

Keywords: Domain Modelling, Graph Databases, Java Frameworks, MVC

JEL classification: D83

DOI: $10.12948 / \mathrm{ie} 2019.03 .10$

\section{Introduction}

In general, knowledge-driven information systems require frequent updates in both their structure and content, because knowledge is unpredictable in its evolution - i.e., new relationships may emerge as frequently as new instance data/facts are added. This is the case for systems in fields such as medicine, sensor-based applications, or social networking [1]. We face a productivity problem in the engineering of such systems, because traditional relational databases offer rather rigidly structured repositories. Such systems must be re-engineered every time we need to accommodate new relationships, and these changes will affect the whole stack of application layers up to the GUI seen by the end-user.

This article proposes a graph-driven approach for managing schema-independent information systems. We deploy a prototype for an academic management system, demonstrating how we can model the scientific output of our Research Center and present it in a friendly GUI that facilitates data navigation regardless of the data schema. The purpose of our undertaking is to prove that we can deploy a knowledge-based information system robust to software engineering changes, even if the underlying data is volatile in both content and structure. In the 
traditional setups employing SQL data stores, any change request on the model structure propagates through the entire development stack of the application - starting from the data layer, through the middleware with object-relational mapped entities and affecting even the Web presentation layer. Thus, we propose a data-modelling solution to this "snowball of management complexity" problem.

In this paper, we also advance previous work [2] on building academic management systems, by replacing the RDF graph with a label property graph (LPG) model.

This enables expanding further our modelling domain capabilities by bridging the gap between the "whiteboard" model and the actual database model [3]. Benefitting from the LPG, we design as a proof of the concept an automated data browsing system that enables traversal of a resource's neighbours, agnostic of their type and the semantics of its connections.

The paper is structured as following: Section 2 shortly introduces related work. Section 3 highlights the methodologies employed within our prototype development and streamlines our contribution, presenting the major capabilities of prototype. Section 4 provides technical details, including an implementation-ready stack for powering the graph DB. Section 5 discusses the main findings, pointing out the strengths and weaknesses of our approach. Last section concludes the paper.

\section{Background and related work}

Both SQL and graph databases use relationships in modelling the data, but SQL data management systems are "data-centric" - relationships being added as a workaround during normalization, while graph databases are coined as "relationship-centric" - with data accumulating around the nodes in an ever-expanding network of relationships [9]. As we need to deal with data of an unpredictable schema, we concluded that a focus-shift from viewing the data as a "small set of data described by a fixed schema" [10] to an "ever-evolving scalable net of resources" [11] is necessary.

The related RDF approach for knowledge modelling gives the possibility that queries related to academic knowledge traverse from the local store to open graphs such as Springer's, effectively linking personal repository resources to the ever-evolving and promising Web of Data [12], benefiting from the RDF standard [13]. In our approach, by using the alternative LPG model, we can distinguish the relationship properties from the nodes' properties, succeeding to shift the load from resources / nouns to the actual relationships / predicates.

Our view of an ever-evolving knowledge repository has already been captured in [11], where it was applied for language resources. In this paper, we model our Research Center's scientific output. The workflow of [11] inspired us, since their database was also gradually expanding thus, incomplete. By rendering links on the view layer to related knowledge, we automate knowledge navigation in a similar fashion to what the Web permitted for documents in the age of Hypertext [14].

\section{Research method and prototype summary}

From a software engineering point of view, we followed the Extreme Programming model to design and implement our prototype. We guided our research effort according with the Action Research methodology [4] based on actual needs identified in our institution. Each iteration of our prototype led to reflections on its results and was used to generate further insight. This methodology helped us diagnose the problems that would arise if representing incomplete knowledge using traditional SQL-based systems, leading us to the solution of using graph databases. Furthermore, the conclusions at each step led to solving the issues related to the development our system, simultaneously producing guidelines [4] for projects that use data of unpredictable or unstable schema. 
We started the software engineering cycle focusing on representing data in a relationshipcentric manner, which resulted in a Neo4 $\mathrm{j}$ [15] store. This successively led to changes in ways of how we represented the Research Center's publications in relationship to the type of collections they appeared in. Ultimately, we developed a module to navigate the knowledge base in a continuous fashion.

Figure 1 shows the architecture of our proposed knowledge-driven academic management system. The starting point is capturing knowledge from the application domain into an entity structure in the database. A concept like the relationship between a paper and the proceedings it has appeared in is effectively inserted in the Neo4j Graph. This scenario is pursued mainly by the SysAdmin and highlighted in the picture with actions denoted by I, II, and so on.

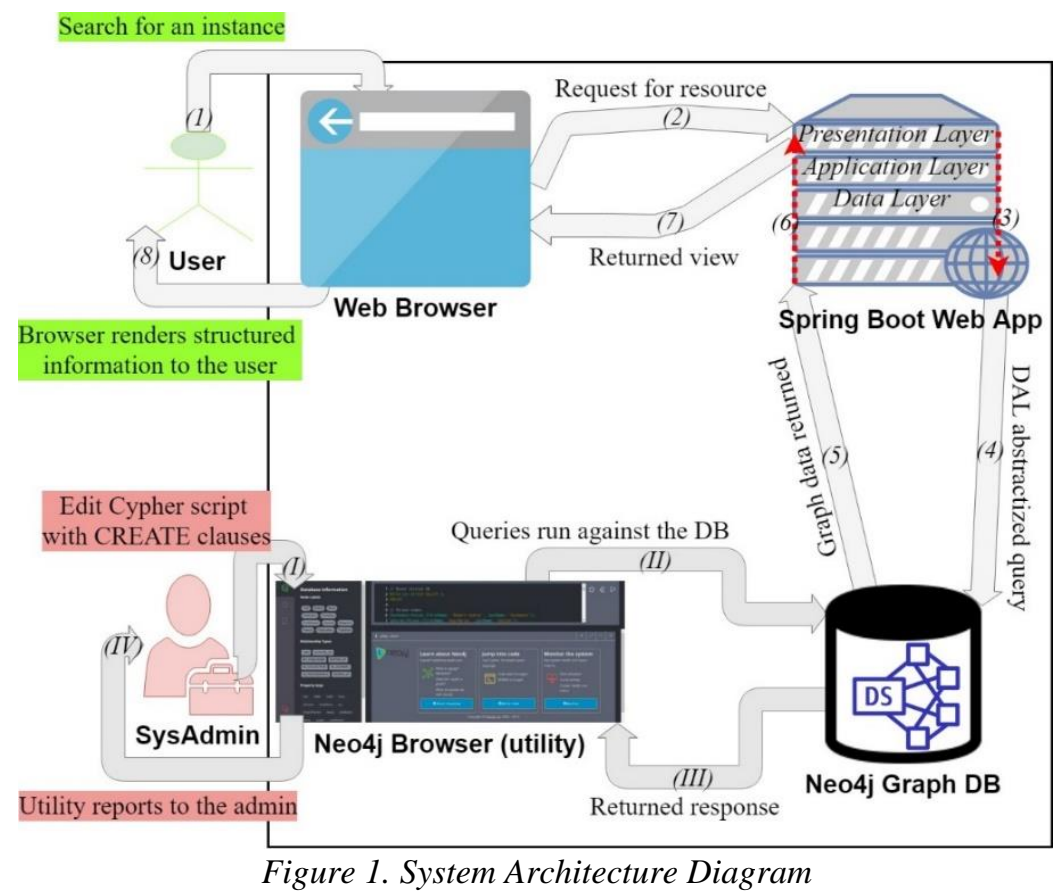

The Middleware consists of a Spring Boot Java Framework [7] implementation of a classic MVC architecture. A typical use case consists of the user requesting a resource via HTTP; the request gets mapped to a controller method, calling a specific service implementation tied to a Data Access Layer (DAL) instance. The returned result is constructed into a model and rendered to the front-end using the Thymeleaf View Technology. To bring the Neo4J data in the model of the Spring Boot framework, we defined related POJO classes for each node type from Neo4J.

With this we obtain a layered architecture which ensures separation of concerns between the presentation, business logic and data persistence layer. We depict this in the upper-right corner box of Figure 1.

\section{Design and implementation details}

Knowledge related to our Research Center activities is stored in Neo4j, being created and queried using the proprietary Cypher query language [5]. Using a central script, the SysAdmin creates the entities, inserts or merges them, if needed, all in the same CREATE statement - as opposed to SQL, were schema/relation definition and actual population with records have separate statements. As a result, restructuring the data and the definition of instances with unforeseen properties is less restricting than within a system with a fixed a schema definition as a prerequisite. 
Since Neo4j engine generates an id for each node / relation at creation (see the bottom of Figure 2 ), any resource stored can be retrieved with minimum delay. For retrieving neighbours of a node, Neo4j [6] uses index-free adjacency. The engine ensures that every node has pointers to neighbours, powered by a native-graph engine. The fact that the information is actually stored in graph data structures dramatically improves the processing speed [6].

Using Spring Boot [7], we managed to create a universal endpoint that activates whenever a request is made to its URL that is suffixed with / and a valid id from the DB. A valid use case would consist of requesting information about the node associated with a member of our Research Center, retrieving all related content, including the contributed publications. In the provided example (depicted in Figure 2), we ran a manual query (at the top of the figure) for a subgraph with all the books and their $1^{\text {st }}$ level neighbours. From the subset of all the retrieved nodes (displayed at the middle of the figure), we identified two books (with meta information depicted at the bottom of the figure), one with the DOI property and the other missing it. We can notice that instances of a particular node type ("label", in Neo4J) are not necessarily compliant to the same prescribed schema. Accessing the book resource through a request with the node id, relaunches a request that ends in displaying information about that current resource in the graph, along with the neighbours (that are back-linked to that same universal endpoint, but using their own id).

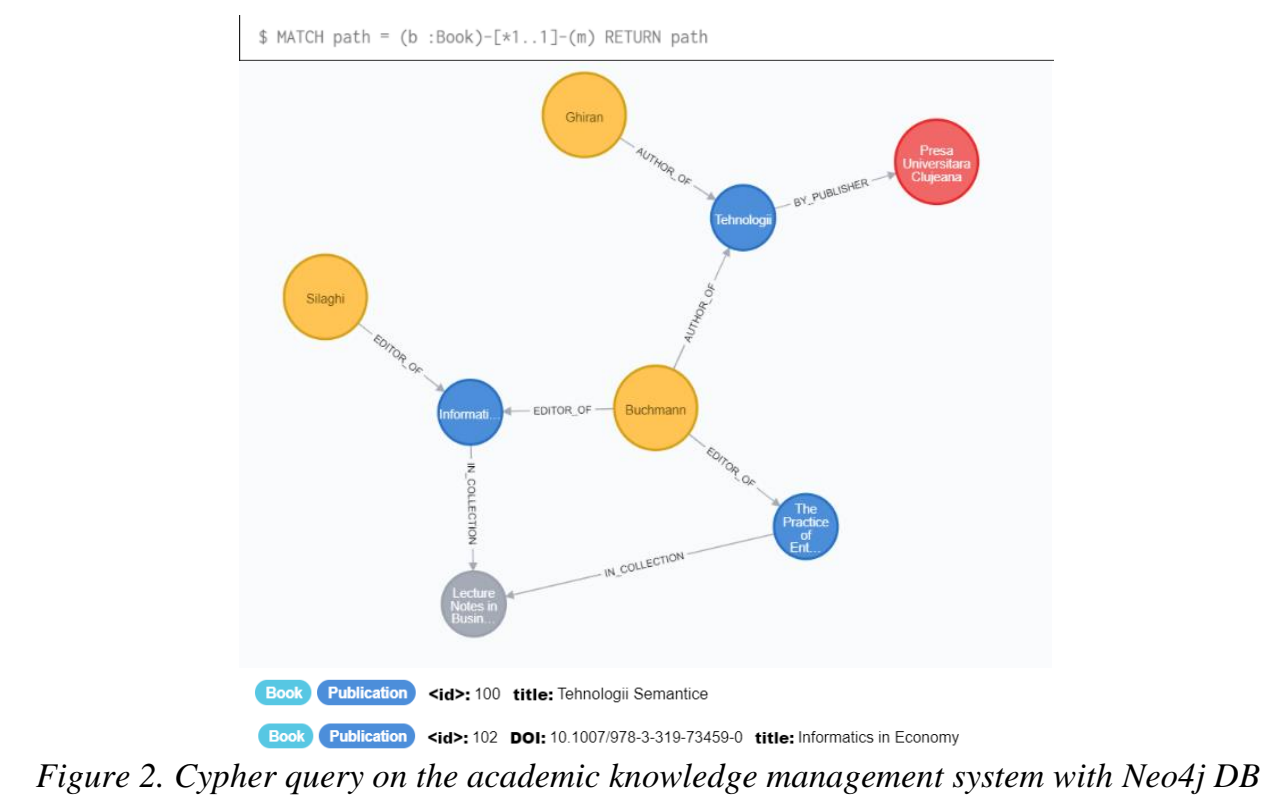

This intermediary mapping from instance resources to full-fledged Java objects is done underthe-hood by the Spring Data Neo4j (SDN [8]) implementation over the Object Graph Mapper. Without touching the technical jargon in too much detail, we linked our application with the database-end using the latest SDN dependency. This provided us with generic CRUD operations, which were generated at runtime for a specific class. We used these with our endpoints, where particular classes are referenced in the View layer, thus benefitting from the existing findAll() method tied to the specific Class type. SDN automatically creates a query under-the-hood and communicates with Neo4j using the ultra-fast Bolt protocol. We managed to "exploit" these generic repositories to extend a pool of objects whose type is not given at runtime, and make use of the alternate written-query methods thanks to the @ Query annotation. Thus, we write Cypher queries with customized logic directly within the Repository classes of the Spring architecture. Both results are shown in Listing 1, as service methods that take an ID 
as parameter and use it inside the query to retrieve information about the requested node and nodes that touch it.

Listing 1. Neo4j Repository derived interface; uses hardcoded Cypher queries and return lists with return parameters name-object pairs

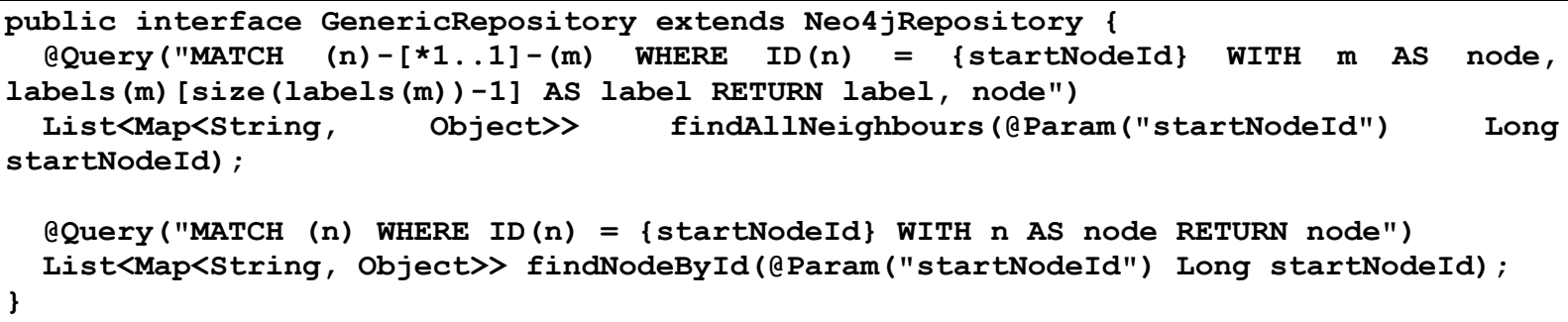

\section{Results and discussion}

The benefit of implementing the prototype in this way is perceived in terms of responsiveness i.e. time taken to return a resource's description, as well as structural flexibility.

Neo4j enables rapid prototyping of knowledge graphs thanks to a straightforward model: we can correlate the notions of an LPG with Language Syntax [15]: Nodes (nouns) have properties (adjectives) and are in relationships (verbs) that also have properties (adverbs), doing something to other Nodes (nouns/objects). This ability to describe a directed relationship by additional properties adds more semantics to the knowledge that is intrinsically contained, providing for a quick query mechanism since such an edge is also treated as a Neo4j entity (like nodes).

Abstracting interactions with graph data is done through Spring Boot, with the advantage of the framework providing a common workflow for general MVC application development. Thanks to the integration with $\mathrm{Neo} 4 \mathrm{j}$, the DAL code strictly resides to functionalities about the domain classes and hides configuration details. Learning that instances of the root Object class returned from the DAL retain domain-specific class identity, led us to designing an intuitive algorithm. Through the use of RTTI we managed to evenly distribute objects of a particular class (the equivalent of node instances with a definite label) across lists in the model, reducing the controller methods to a single one that handles any kind of resource. As a result, we managed to move some "load" of the stack from the back-end to the presentation layer, where we exposed different views for each category of selected node.

The strengths of our solution are that it provides guidelines for the design and development of a relationship-centric information systems. Being graph-driven, usage of a schema is not prescribed, thus allowing for easy prototyping of data models. For the selected use case presented in section 4, new scientists can easily be integrated in the existing graph, and books that do not have a DOI simply could miss that property, saving space comparing to a traditional approach where a null value must be assigned. Further development of complex business logic on the API end is facilitated thanks to the decoupling of concerns, achieved by following principles stated in [16], ultimately enabling the project to evolve in further directions without changing parts of the code, but by rather reusing them.

These advantages come with the cost of several weaknesses. By diverging from the RDF standard, and limiting to in-house CRUD operations written in a script, our solution is a closed, technology-specific one. Refining the graph according to the Linked Data principles would further increase the reach of our local queries, permitting logic-based inference and federation. 
www.conferenceie.ase.ro

\section{Conclusion}

In this paper, we dealt with the concept of representing ever-evolving data and building a knowledge-driven information system on it. We analysed the case of an academic information system and demonstrated a full-stack object-oriented solution with a well-formed architecture. The great asset of our prototype is scalability and flexibility for further changes, due to separating concerns and lack of a preimposed data schema. Neo4j proved to be a reliable database system that took the overhead of normalization away, emphasizing relationshipcentric data modelling and intuitive front-end navigation of data through graph queries.

\section{References}

[1] G. C. Deka, NoSQL: Database for Storage and Retrieval of Data in Cloud, New York: Chapman and Hall/CRC, 2017.

[2] L. Mocean and R. A. Buchmann, "Open Knowledge-aware Academic Management Systems", in Proc. of the 18th European Conference on Knowledge Management, Barcelona, Spain, 2017, Academic Conferences and Publishing International Limited, p. 714-722, 2017.

[3] I. Robinson, J. Webber and E. Eifrem, Graph Databases: New Opportunities for Connected Data, 2nd edition, O'Reilly Media, 2015.

[4] M. Denscombe, The Good Research Guide: for small-scale social research projects, 4th ed., Berkshire: Open University Press, 2010.

[5] Neo4j, Inc., "Cypher Query Language". [Online]. Available: https://neo4j.com/developer/cypher. [Accessed March, 2019].

[6] Neo4j Inc., "Neo4j Graph Platform". [Online]. Available: https://neo4j.com. [Accessed March, 2019].

[7] Pivotal Software, Inc., "Spring Boot”. [Online]. Available: https://spring.io/projects/springboot. [Accessed March, 2019].

[8] Pivotal Software, Inc., "Spring Data Neo4j”. [Online]. Available: https://spring.io/projects/spring-data-neo4j. [Accessed March, 2019].

[9] D. Karagiannis and R. A. Buchmann, "A Proposal for Deploying Hybrid Knowledge Bases: the ADOxx-to-GraphDB Interoperability Case," in Proc. of the 51st Hawaii Intl. Conf. on System Sciences, Hawaii, USA, p. 4055-4064, 2018.

[10] S. C. Tamane, N. Dey and V. K. Solanki, Privacy and Security Policies in Big Data, Hershey: IGI Global, 2017.

[11] C. Federmann, I. Giannopoulou, C. Girardi, O. Hamon, D. Mavroeidis, S. Minutoli and M. Schröder, "META-SHARE v2: An Open Network of Repositories for Language Resources including Data and Tools," in Proc. of the 8th Intl. Conf. on Language Resources and Evaluation, European Language Resources Association, Istanbul, p. 3300-3303, 2012.

[12] C. Bizer, J. Lehmann, G. Kobilarov, S. Auer, C. Becker, R. Cyganiak and S. Hellmann, "DBpedia - A Crystallization Point for the Web of Data," Web Semantics: Science, Services and Agents on the World Wide Web, vol. 7, no. 3, pp. 154-165, 2009.

[13] W3C, "RDF - Semantic Web Standards". [Online]. Available: https://www.w3.org/RDF/ [Accessed March, 2019].

[14] T. Berners-Lee, "What the Semantic Web can represent". [Online], 1998. Available: https://www.w3.org/DesignIssues/RDFnot.html

[15] Neo4j Inc., "Introduction to Neo4j". [Online]. Available: https://neo4j.com/graphacademy/online-training/introduction-to-neo4j. [Accessed March, 2019].

[16] R. C. Martin, Agile Software Development: Principles, Patterns, and Practices, New Jersey: Prentice Hall, 2002 